\title{
Tratamento ortopédico da Classe III em padrões faciais distintos
}

Paula Vanessa Pedron Oltramari*, Daniela Gamba Garib**, Ana Cláudia de Castro Ferreira Conti***, José Fernando Castanha Henriques ${ }^{\star \star \star \star}$, Marcos Roberto de Freitas ${ }^{\star \star \star \star}$

\section{Resumo}

A má oclusão de Classe III, de origem essencialmente esquelética, produz uma acentuada deformidade facial. A Classe III pode ser interceptada durante a fase de crescimento e desenvolvimento craniofacial mediante o uso de aparelhos ortopédicos. O crescimento mandibular, predominantemente endocondral na cartilagem condilar, obedece essencialmente ao controle genético. Portanto, os efeitos dos aparelhos ortopédicos que visam restringir o crescimento da mandíbula mostram-se limitados, o que conduz ao pobre prognóstico de tratamento precoce da Classe III determinada pelo prognatismo mandibular. Felizmente, o componente esquelético maxilar responde melhor à aplicação de forças ortopédicas, já que o crescimento ósseo intramembranoso mostra-se mais susceptível a influências extrínsecas ou ambientais. Deste modo, a Classe III morfologicamente definida pelo retrognatismo maxilar, privilegia-se com o tratamento ortopédico. Este trabalho discute o tratamento interceptivo da Classe III por meio da tração reversa da maxila em pacientes com padrões faciais distintos, apresentando dois casos clínicos, e ainda ressalta os aspectos atinentes a sua estabilidade.

Palavras-chave: Máscara facial. Expansão rápida da maxila. Prognatismo esquelético. Classe III.

\section{INTRODUÇÃO}

O tratamento precoce da má oclusão de Classe III tem mostrado efetividade do ponto de vista esquelético ${ }^{23,30}$, em especial quando essa má oclusão possui um componente de retrognatismo maxilar ou uma combinação de retrognatismo maxilar e pequeno prognatismo mandibular, o que representa grande parte dos pacientes com esta deformidade $^{3,14}$. Isto porque, uma vez que as su- turas maxilares formam-se, pela ossificação intramembranosa, são mais susceptíveis a influências ambientais quando comparadas à mandíbula, que possui crescimento essencialmente endocondral e, portanto, mais propenso a determinações genéti$\mathrm{cas}^{4}$. Desta forma, a Ortopedia pode atuar mais facilmente sobre o crescimento da maxila, interceptando precocemente a Classe III definida pelo retrognatismo maxilar.

* Mestre e Doutoranda em Ortodontia pela Faculdade de Odontologia de Bauru - USP.

** Mestre e Doutora em Ortodontia pela Faculdade de Odontologia de Bauru - USP; Professora Associada da Disciplina de Ortodontia da Universidade Cidade de São Paulo - UNICID; Coordenadora do curso de especialização em Ortodontia da Prevodonto Centro de Estudos - Rio de Janeiro.

*** Mestre e Doutora em Ortodontia pela Faculdade de Odontologia de Bauru - USP; Professora da disciplina de Ortodontia da Universidade Paulista - UNIP, Campus Bauru.

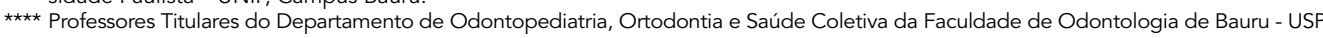




\section{CONSIDERAÇÕES SOBRE O DIAGNÓSTICO}

O diagnóstico ideal da má oclusão de Classe III deve ser precoce, se possível ainda na dentadura decídua. Quanto mais cedo, a interceptação suscita maiores efeitos ortopédicos em detrimento dos inevitáveis efeitos ortodônticos ${ }^{11}$. Além disso, devolver a estética à criança precocemente implica contribuir para sua auto-estima, levando-se em consideração o fator psicológico ${ }^{18}$.

A má oclusão de Classe III agrava-se ao longo do crescimento, principalmente a partir da adolescência. Deste modo, na criança, esta má oclusão não se apresenta totalmente definida, e as características faciais e oclusais ainda sutis podem dificultar o diagnóstico. A identificação precoce desta discrepância esquelética depende da observação minuciosa de uma série de características faciais, oclusais, e cefalométricas que revelam forte tendência à Classe III. A ausência da proeminência zigomática ou malar constitui o sinal facial que traduz o retrognatismo maxilar (Fig. 1.2, 2.2). A linha mento-pescoço aumentada em relação à profundidade da face média e o ângulo mais agudo entre mento e pescoço denunciam a contribuição da mandíbula na Classe III (caso 2).

As características oclusais da Classe III nas dentaduras decídua e mista parecem bastante variáveis. No caso 1, a paciente apresenta-se no primeiro período transitório da dentadura mista, com mordida cruzada total. No caso 2, a mordida cruzada total apresenta-se associada à mordida aberta devido ao hábito persistente de sucção (Fig. 2.3). Mesmo diante da relação canino de Classe I, podemos classificar a má oclusão como Classe III, baseando-se sobretudo no padrão facial, na relação interarcos e secundariamente nas características cefalométricas.

A observação radiográfica constitui um complemento para a análise clínica. $\mathrm{Na}$ radiografia panorâmica, verifica-se a presença de todos os germes dos dentes permanentes não irrompidos e a ausência de distúrbios de irrupção ou patologias. A telerradiografia em norma lateral deve ser avaliada morfologicamente. Os números cefalométricos devem ser considerados com certa cautela, porque pacientes Classe III freqüentemente apresentam a base do crânio encurtada. Com o ponto "N" localizado mais posteriormente, obtém-se leituras errôneas das medidas cefalométricas que utilizam a base do crânio como referência (SNA, Nperp-A, SNB, Nperp-P). A conduta mais coerente para estes pacientes é a utilização de medidas que comparam o comprimento efetivo da maxila (Co-A) e da mandíbula (Co-Gn) com os valores dados no Atlas de Crescimento Craniofacial ${ }^{15}$. No caso 2, os valores iniciais para as medidas Co-A (78mm) e Co-Gn (98mm), quando comparados às medidas de normalidade para a idade Co-A $(81,5 \mathrm{~mm})$ e Co-Gn $(99,3 \mathrm{~mm})$, demonstram a maior participação da maxila que da mandíbula na composição da Classe III. A altura facial ântero-inferior aumentada $60 \mathrm{~mm}$, quando se esperava $58 \mathrm{~mm}$, segundo a tabela de McNamara), juntamente com a inclinação do plano mandibular aumentado indicam, ainda, um predomínio de crescimento vertical neste paciente. A identificação do prognatismo mandibular e do padrão vertical de crescimento empobrecem o prognóstico de tratamento precoce, devido a menores chances de estabilidade pós-tratamento. No caso 1, as medidas iniciais para Co-A $(75 \mathrm{~mm})$ e Co-Gn (98mm) mostram também a maior participação da maxila na determinação da Classe III, já que o Atlas de Crescimento mostra valores de normalidade de $78,9 \mathrm{~mm}$ para a maxila e $95,5 \mathrm{~mm}$ para a mandíbula na idade de 6 anos. Porém, o padrão de crescimento horizontal (SN.GoGn: $33^{\circ} \mathrm{e}$ AFAI: $62 \mathrm{~mm}$ ) contribui na definição de um bom prognóstico de tratamento ortopédico.

O tratamento precoce da má oclusão de Classe III direciona-se para a maxila, por meio da expansão rápida da maxila (ERM) seguida pela protração maxilar ${ }^{2,6,8,9,11,12,23}$. A ERM, além de corrigir a mordida cruzada posterior, estimula a atividade celular das suturas, potencializando os resultados da protração ${ }^{13,19,27,28,29,30}$. 

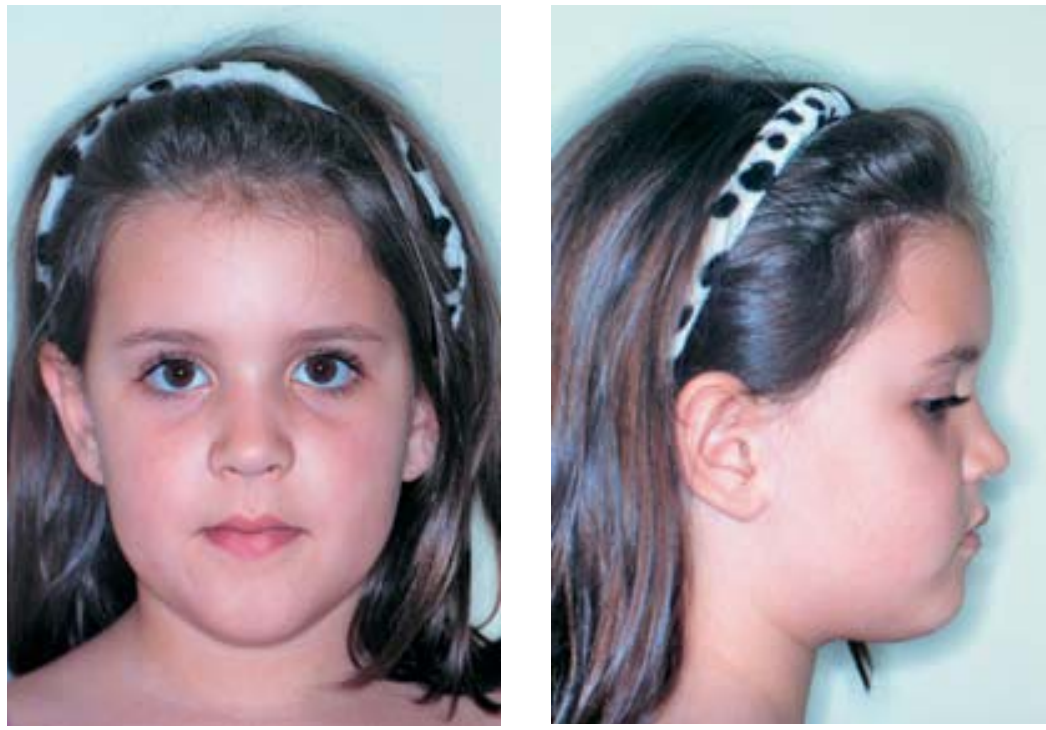

FIGURAS 1.1, 1.2 - Fotos da paciente pré-tratamento frente e perfil.
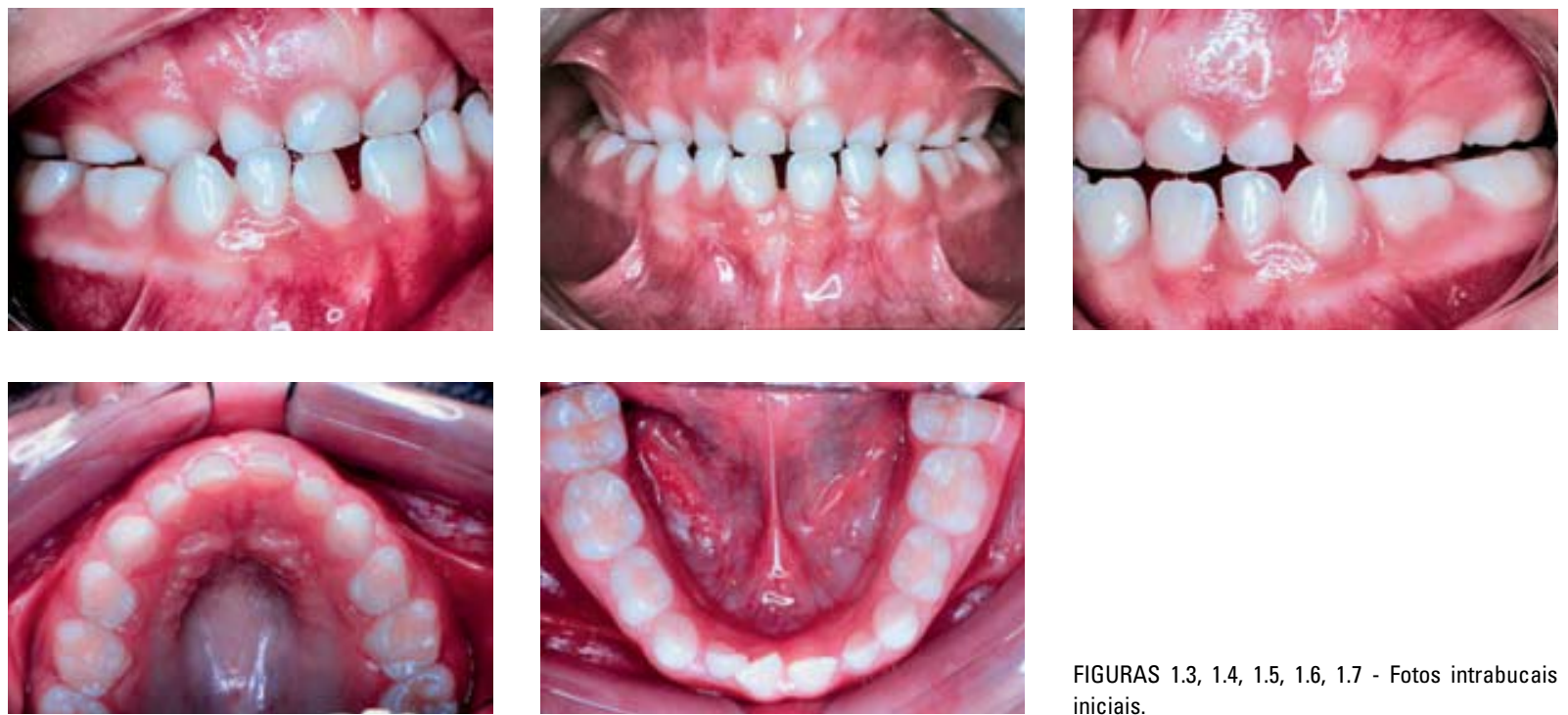

FIGURAS 1.3, 1.4, 1.5, 1.6, 1.7 - Fotos intrabucais iniciais.
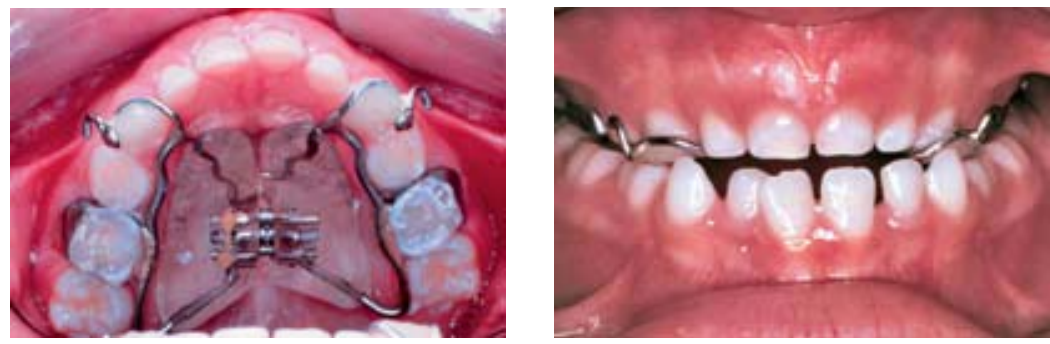

FIGURAS 1.8, 1.9 - Pré-expansão rápida da maxila. 

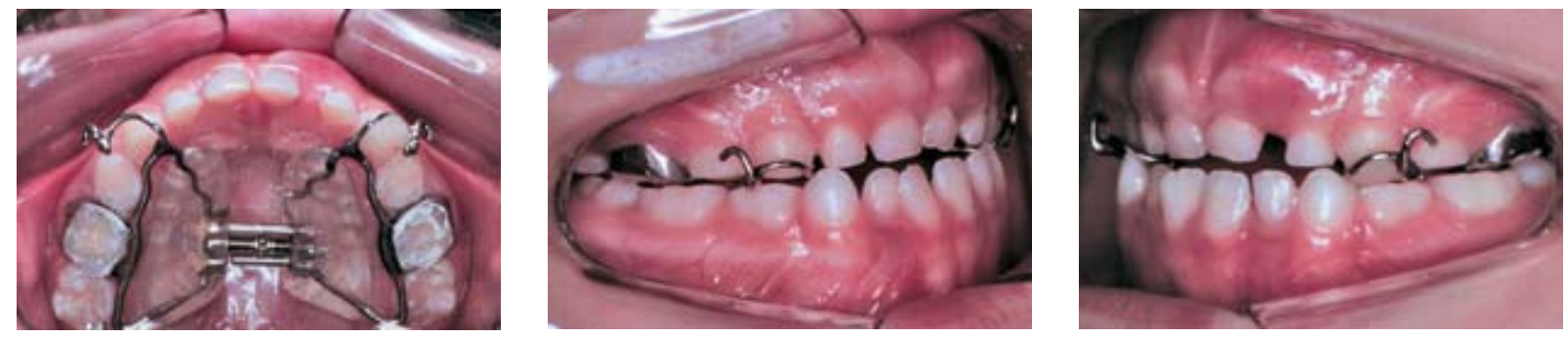

FIGURAS 1.10, 1.11, 1.12 - Pós-expansão rápida da maxila.

FIGURAS 1.13, 1.14 - Instalação da máscara de tração reversa da maxila.
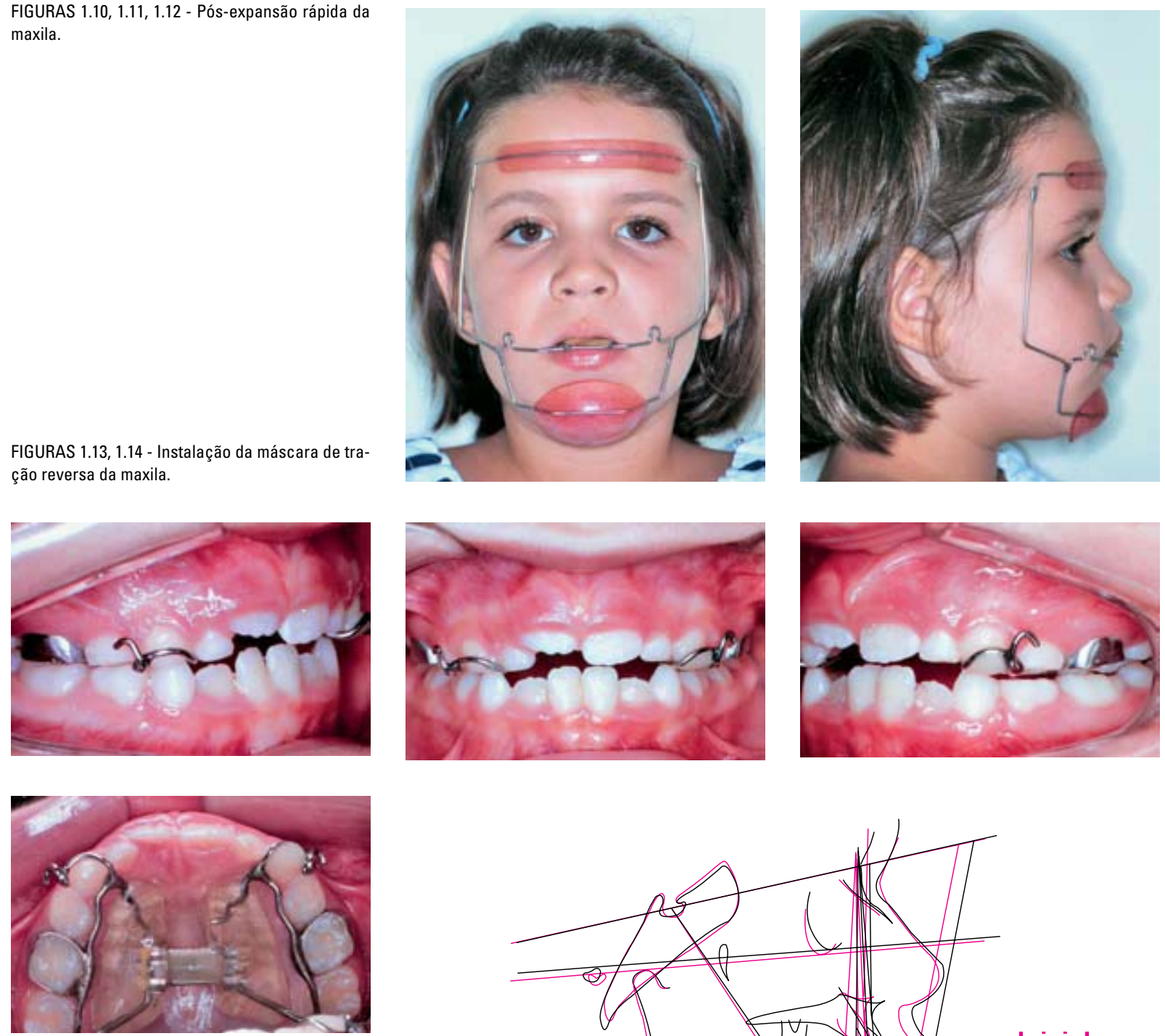

FIGURAS 1.15, 1.16, 1.17, 1.18 - Pós-fase ativa da máscara.

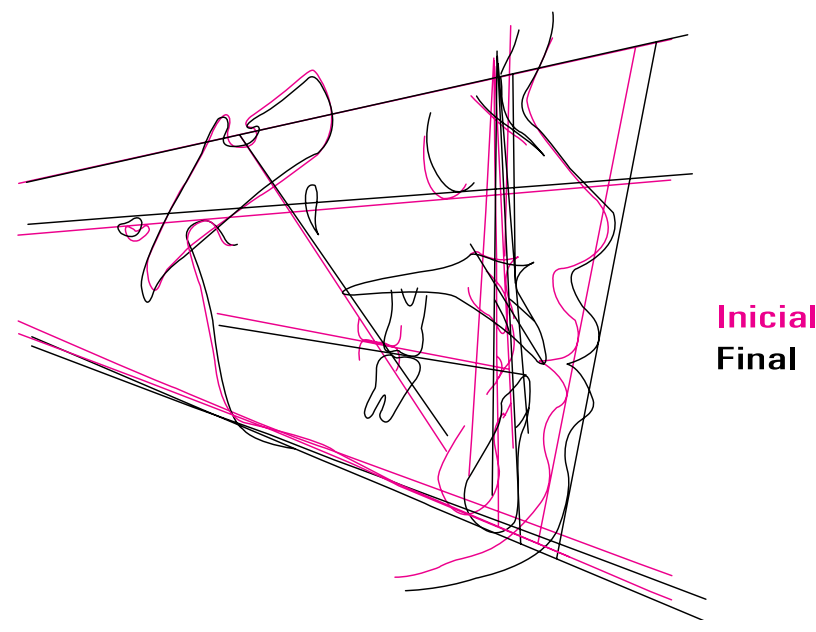

FIGURA 1.19 - Superposição dos cefalogramas inicial e final. 

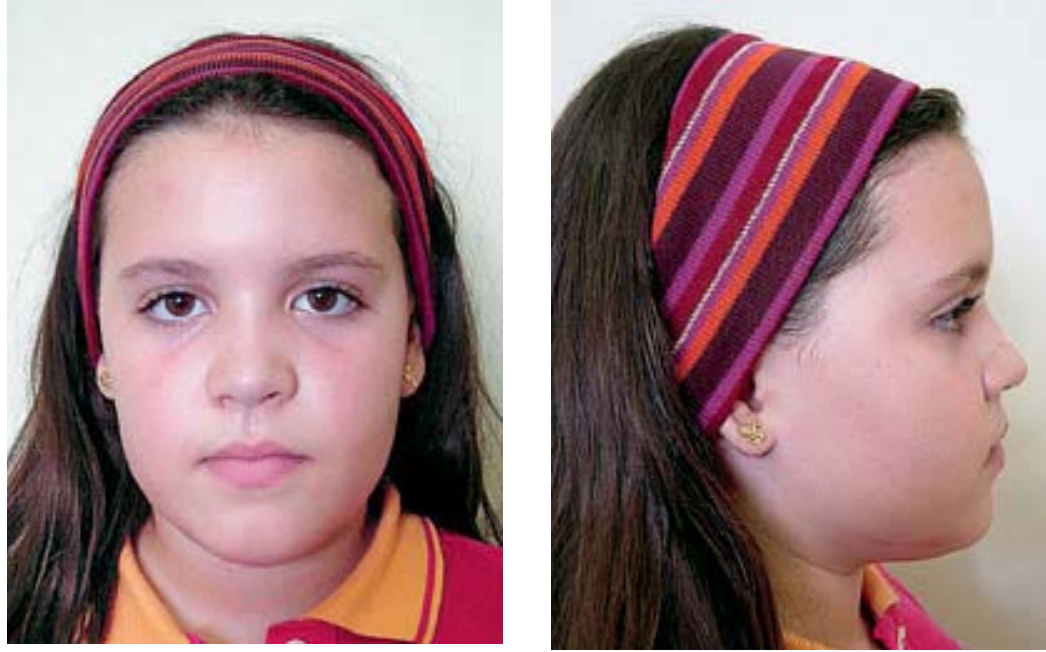

FIGURAS 1.20, 1.21 - Fotos finais frente e perfil.
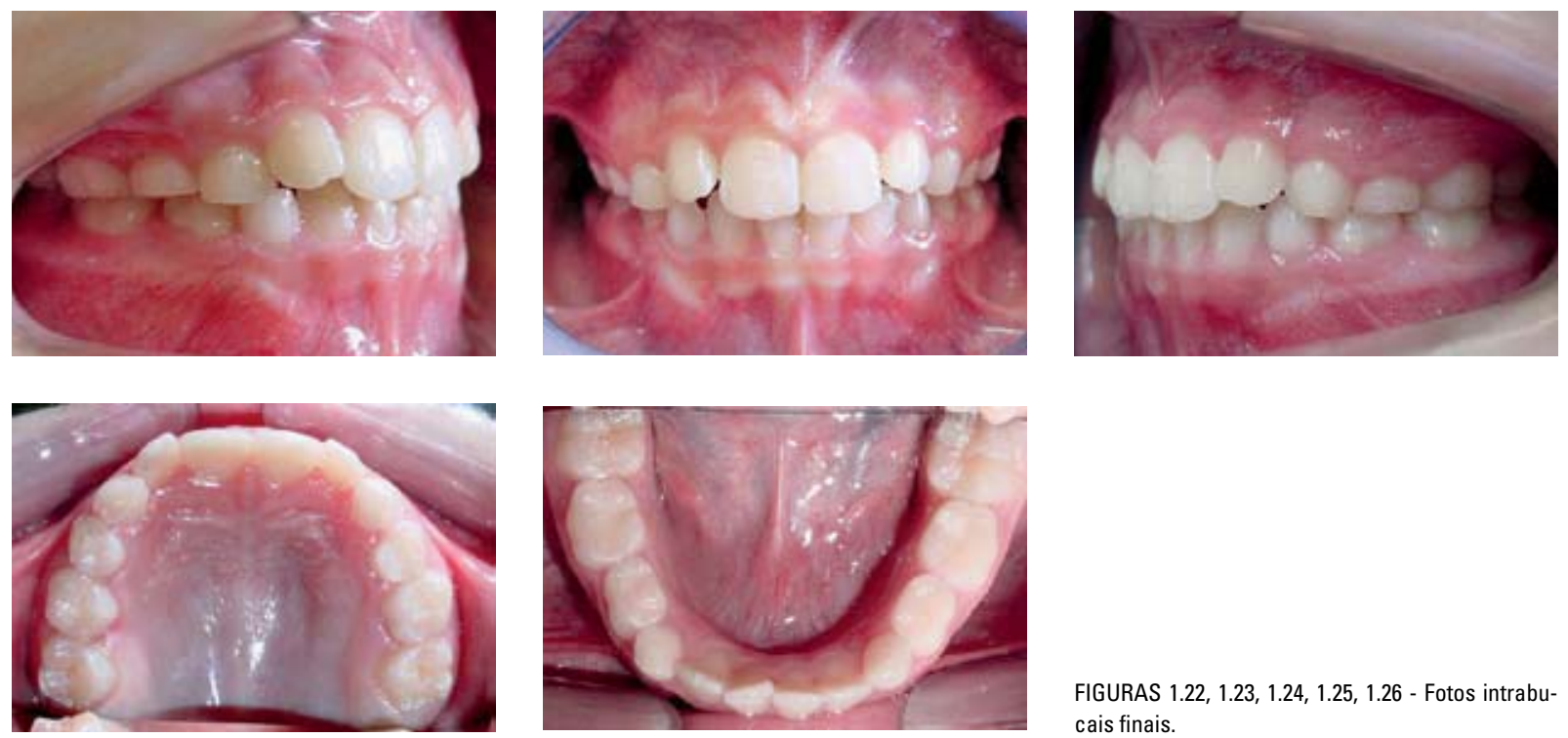

FIGURAS $1.22,1.23,1.24,1.25,1.26$ - Fotos intrabucais finais.
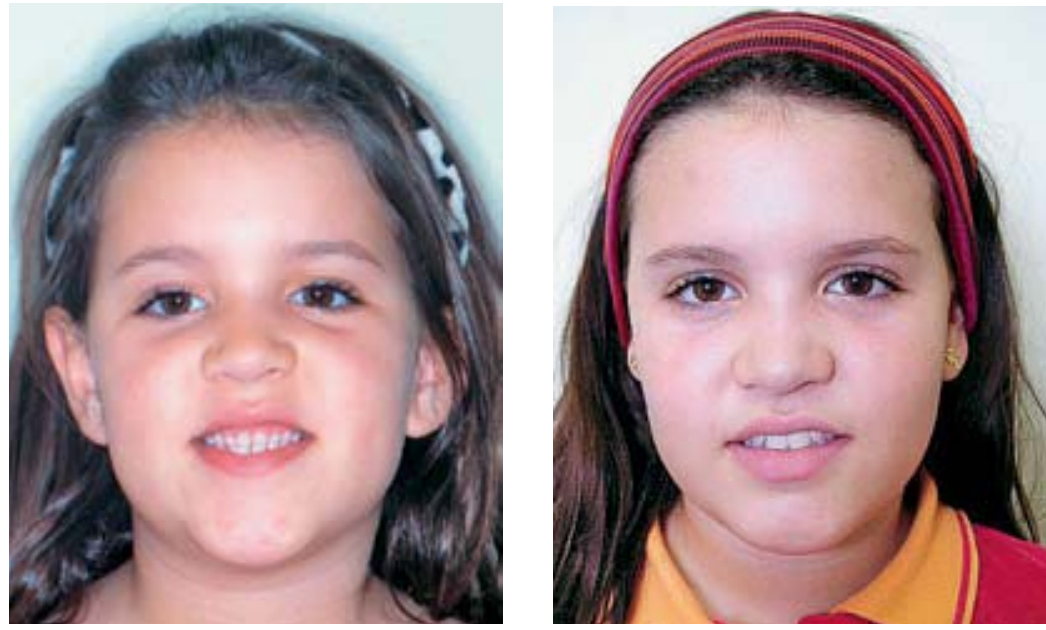

FIGURAS 1.27, 1.28 - Fotos pré e pós-tratamento. 

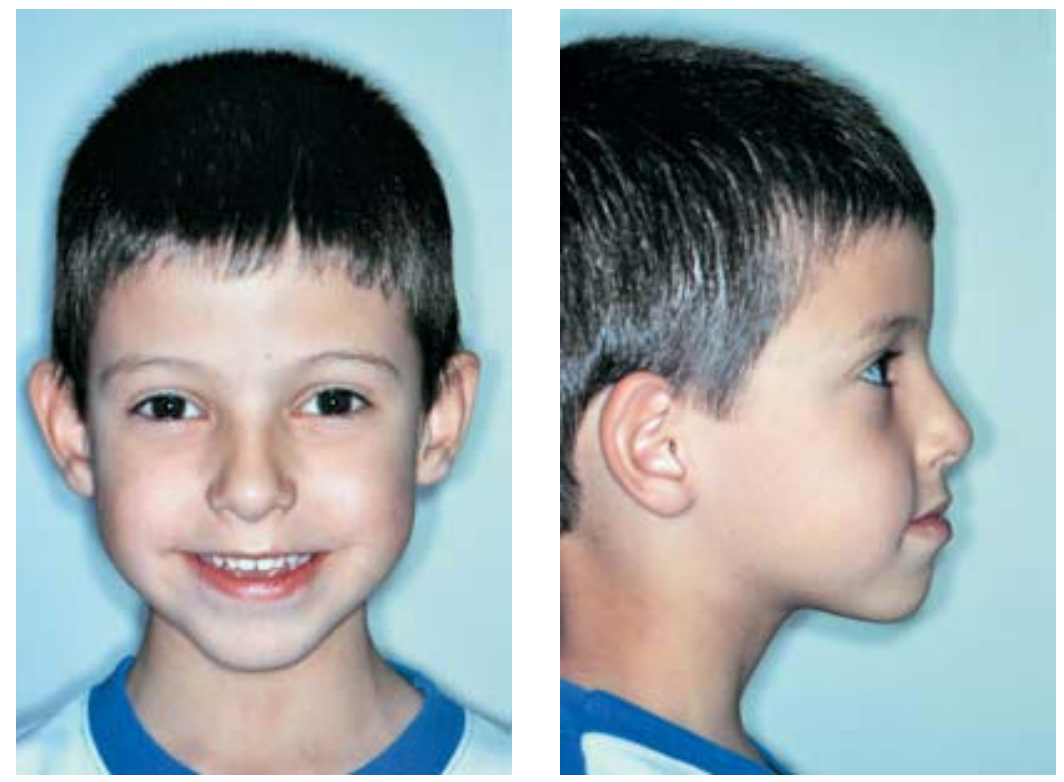

FIGURAS 2.1, 2.2 - Fotos faciais iniciais.
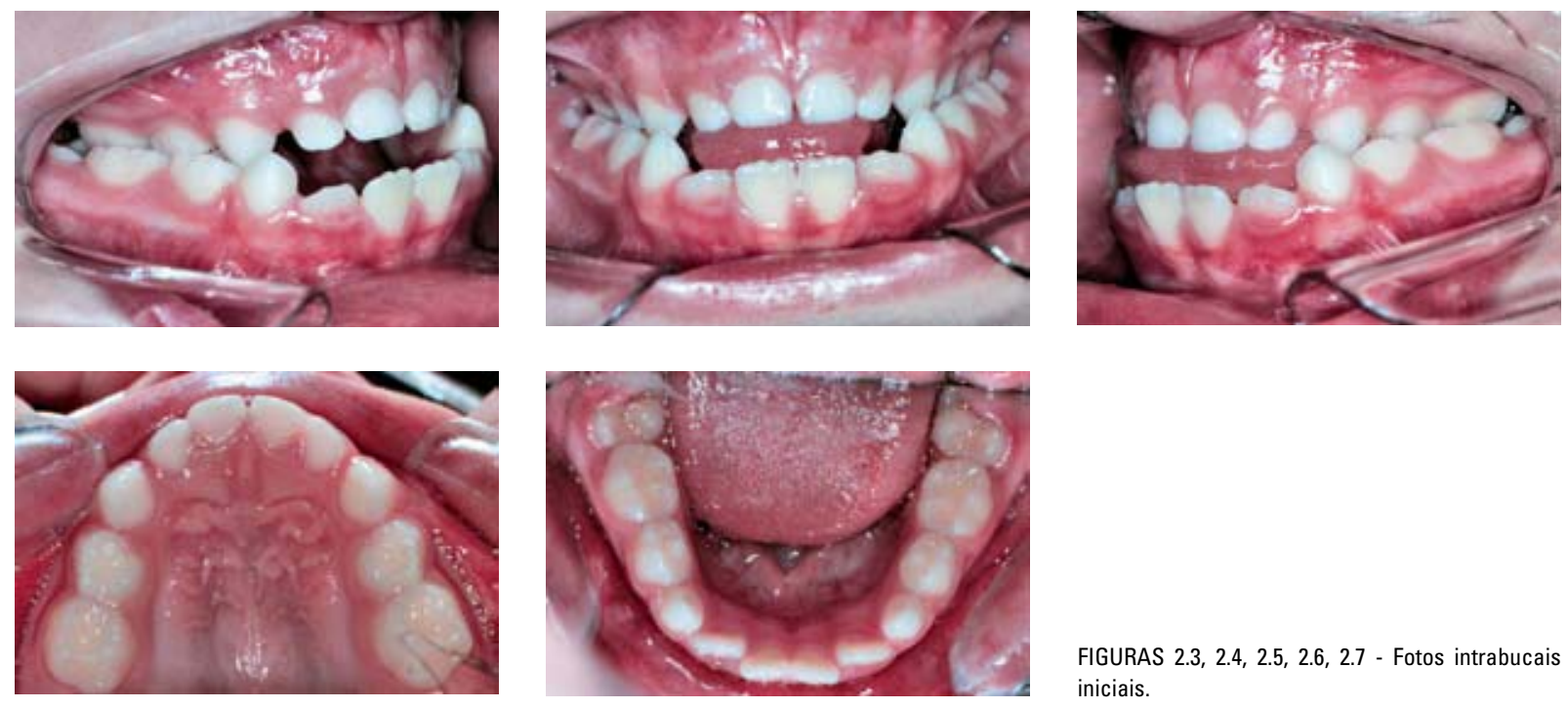

FIGURAS 2.3, 2.4, 2.5, 2.6, 2.7 - Fotos intrabucais iniciais.
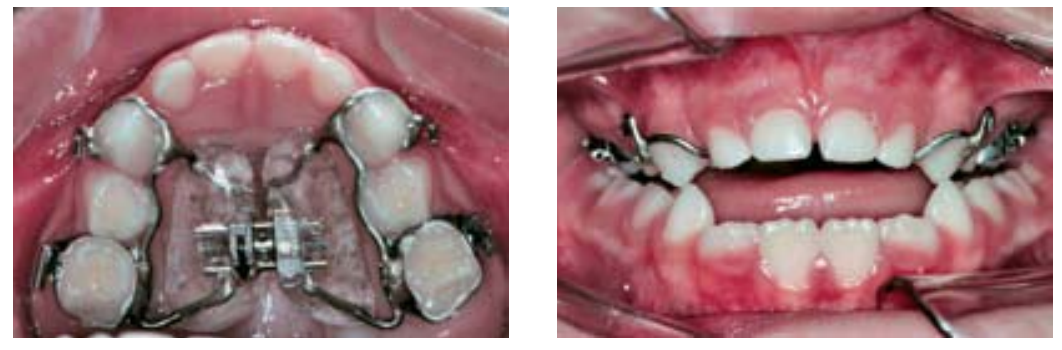

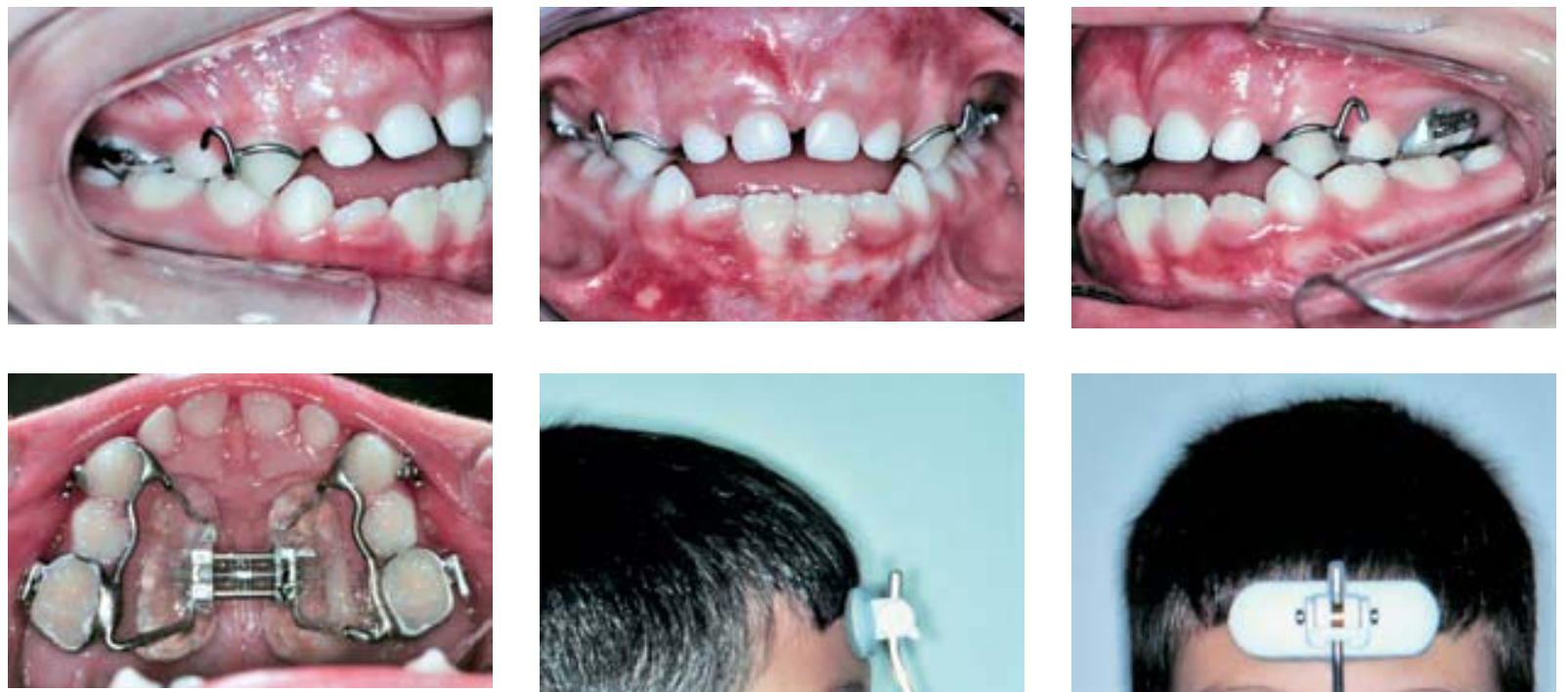

FIGURAS 2.10, 2.11, 2.12, 2.13 - Pós-expansão rápida da maxila.

FIGURAS 2.14, 2.15 - Instalação da máscara de tração reversa da maxila.
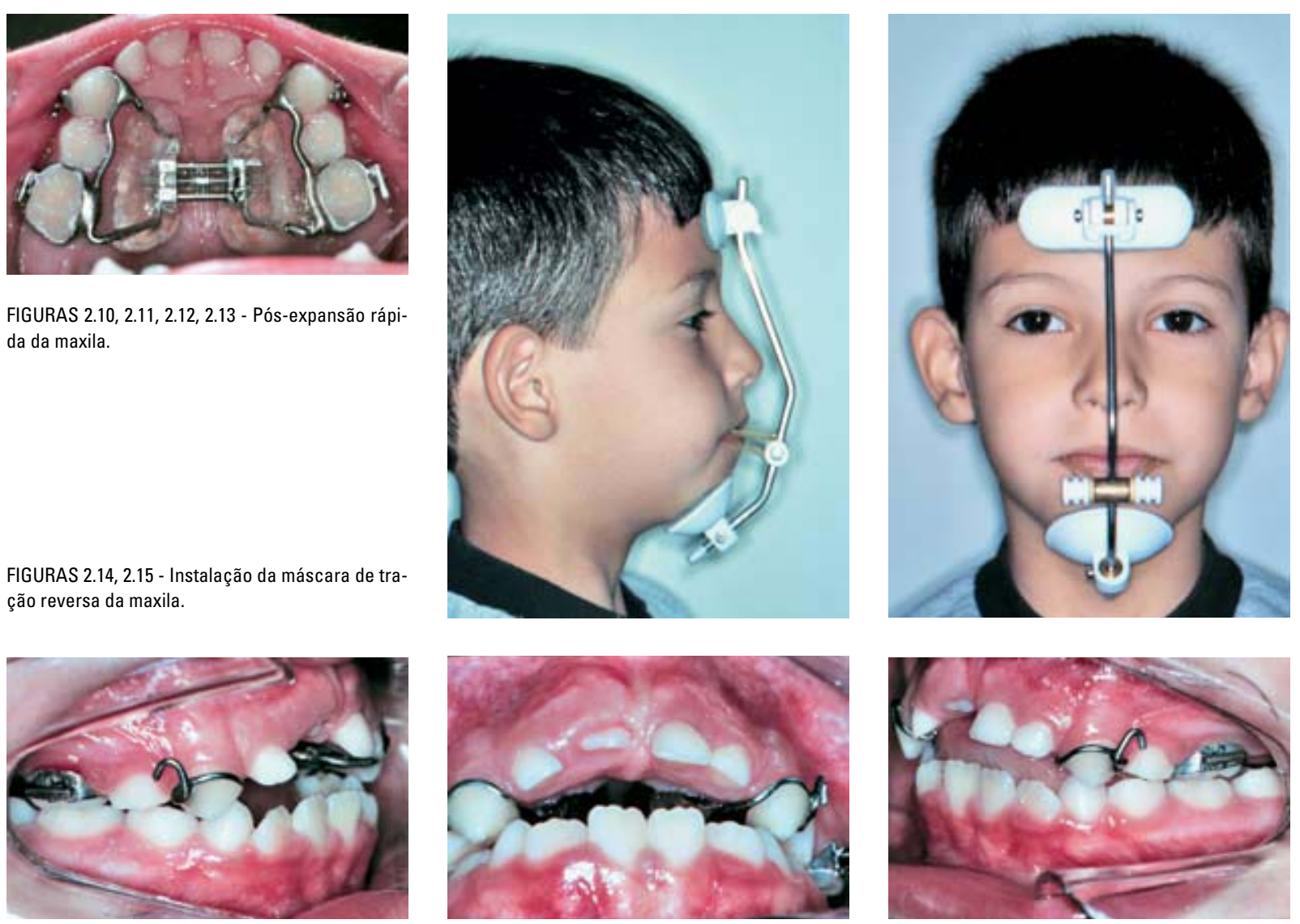

FIGURAS 2.16, 2.17, 2.18 - Pós-fase ativa da máscara.

A ERM pode ser realizada utilizando-se o aparelho expansor tipo Haas ${ }^{20,21,24}$; com ganchos laterais soldados por vestibular para receber os elásticos de protração (Fig. 1.8, 1.9, 2.8, 2.9). A ativação do parafuso obedece ao protocolo de $\mathrm{Haas}^{7}$, extendendo-se aproximadamente por duas semanas. Quando se observa a abertura do diastema interincisivos, por volta do $4^{\circ}$ dia de expansão, instalase a máscara facial para tração reversa da maxila.
Nota-se que a protração maxilar inicia-se ainda durante a fase ativa da ERM ${ }^{1}$. O paciente é orientado a utilizá-la 14 horas/dia, excluindo o período escolar. Há no mercado uma grande variabilidade de aparelhos extrabucais de protração maxilar. No caso 1 optou-se pela máscara facial de Turley, em que é necessária a moldagem facial (Fig. 1.13, 1.14); já no caso 2 , optou-se pela máscara pré-fabricada de Petit (Fig. 2.14, 2.15). 


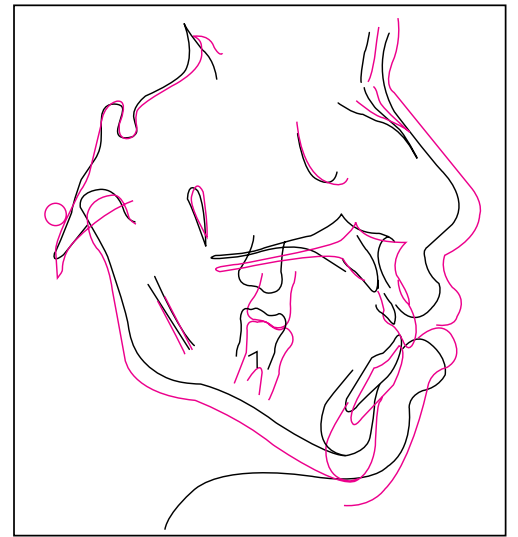

FIGURA 2.19 - Superposição dos cefalogramas inicial e final.

FIGURAS 2.20, 2.21 - Fotos frente e perfil pós-fase ativa da máscara.

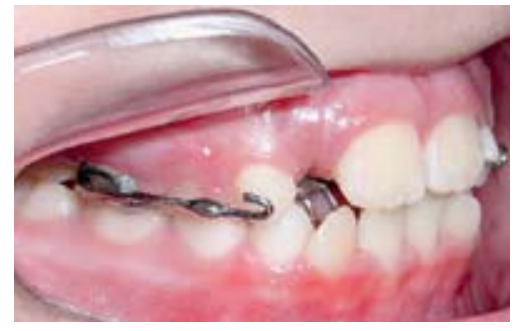

FIGURAS 2.22, 2.23, 2.24, 2.25 - Troca do expansor tipo Haas pelo arco palatino com grade para a fase de contenção da máscara facial, até completar 1 ano de tratamento.
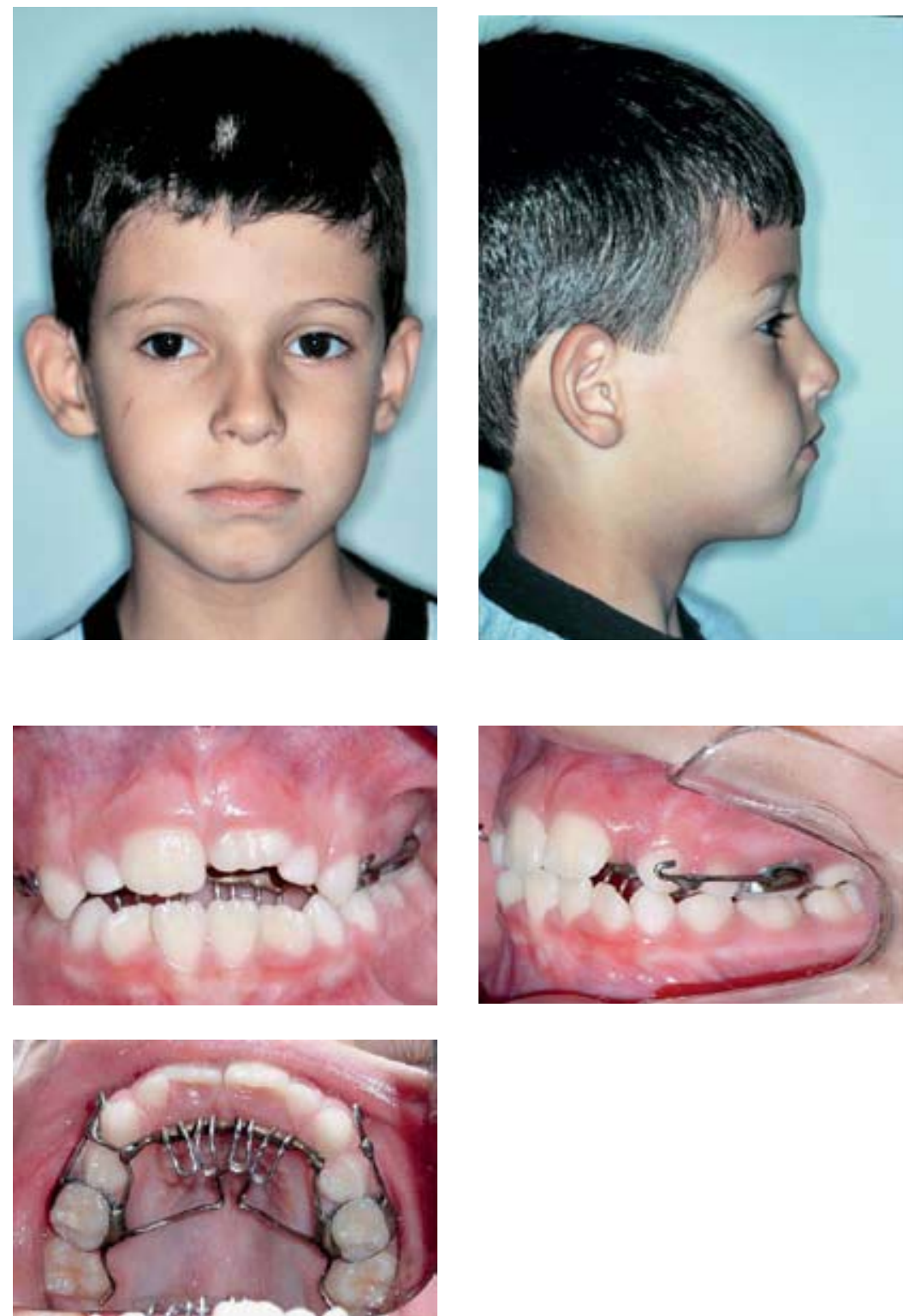

Alcançada a sobrecorreção do trespasse horizontal, a máscara é utilizada como contenção, somente para dormir, pois já se alcançou a sobrecorreção do trespasse horizontal. No caso 2, o aparelho expansor foi substituído por uma barra palatina fixa com grade, para facilitar a higienização e correção da mordida aberta anterior (Fig. 2.22, $2.23,2.24,2.25)$. O estabelecimento do trespasse vertical adequado, antes da remoção da máscara, apresenta grande importância para a estabilidade do tratamento (Fig. 2.16).

Após o uso da máscara de tração reversa da maxila, verificou-se o deslocamento anterior da maxila, atestado pelo aumento do SNA, Nper$\mathrm{pA}$, e a rotação da mandíbula no sentido horário, comprovado pelo aumento do SN.GoGn e redução do $\mathrm{SNB}$, que conjuntamente contribuíram para o aumento da convexidade facial (ANB e NAP) e para a melhora dos contornos faciais. (Fig. 1.19, 2.19). 


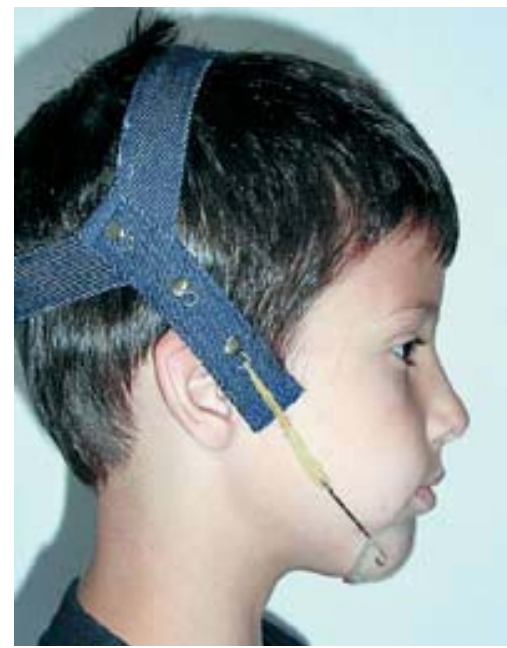

FIGURA 2.26 - Instalação da mentoneira.

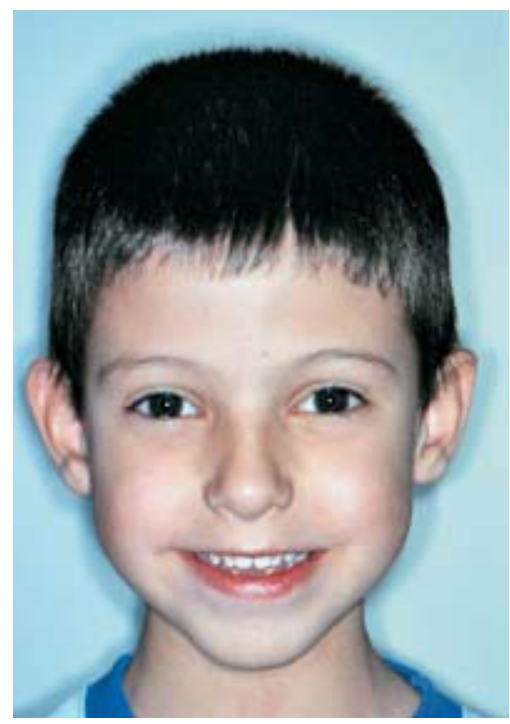

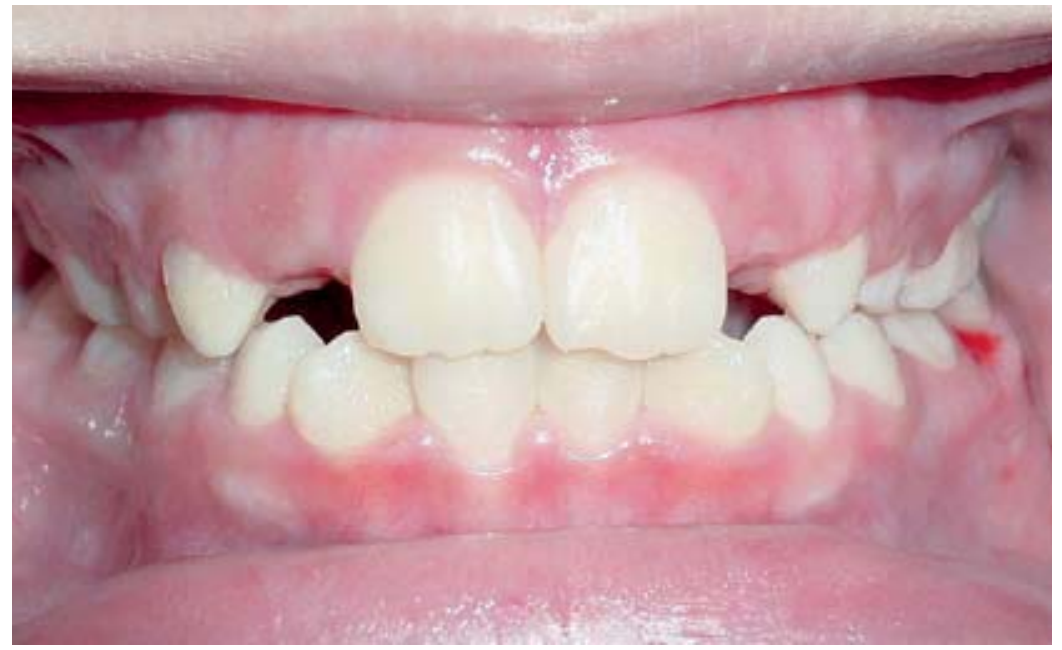

FIGURA 2.27 - Foto intrabucal final.

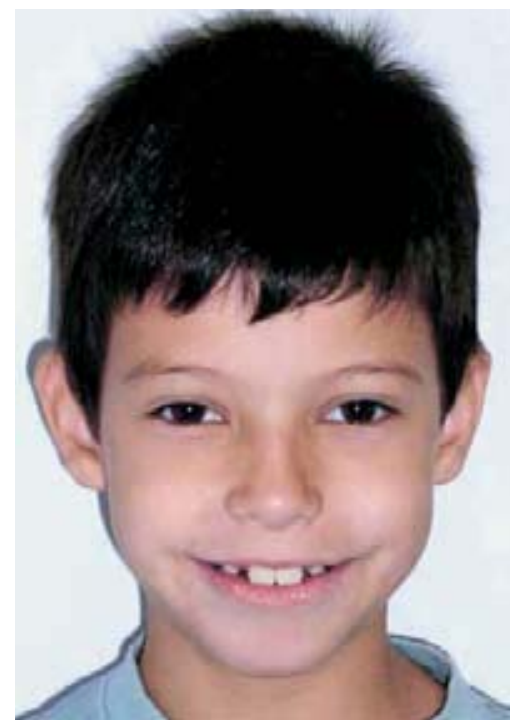

FIGURAS 2.28, 2.29 - Fotos pré e pós-tratamento.
No caso 2 após a suspensão da máscara facial, diante do componente de prognatismo mandibular, prolongou-se a contenção ortopédica por mais um ano, com o uso noturno da mentoneira (Fig. 2.26).

\section{DISCUSSÃO}

Uma vez diagnosticada a Classe III, deve-se considerar a possibilidade de interceptação precoce. Isso possibilita um bom relacionamento oclu- sal, facial e psico-social, favorecendo o crescimento e desenvolvimento normal da criança.

A correção da Classe III esquelética, por meio da expansão rápida aliada à tração reversa da maxila, resulta de uma combinação de mudanças esqueléticas e dentárias que produzem a melhora significante do perfil facial. No entanto, esta opção cabe somente à Classe III com envolvimento maxilar em sua configuração morfológica. Apesar do diagnóstico e tratamento corretos, parece difícil 
prever a estabilidade dessa terapia, uma vez que dependerá do padrão de crescimento do paciente pós-tratamento ${ }^{10}$. Contudo, pode-se realizar um prognóstico positivo ou negativo baseando-se nas características morfológicas iniciais, isto é, considerando-se o componente maxilar ou mandibular que compõe a Classe III, juntamente com o padrão de crescimento facial. No caso 1, a análise facial denunciava uma significante deficiência maxilar com a mandíbula muito aceitável, e a paciente apresenta um padrão de crescimento horizontal, que permitia as alterações verticais compensatórias, inerentes ao tratamento ortopédico. Uma vez que é mais fácil o tratamento e a obtenção da estabilidade sobre o crescimento da maxila, esta paciente apresenta um prognóstico bastante favorável. Portanto, possivelmente apresentará a manutenção dos resultados do tratamento precoce ao longo do desenvolvimento subseqüente. Já no caso 2, o paciente apresenta um padrão de crescimento vertical, e apesar da retrognatismo maxilar, a mandibula apresenta-se com uma marcante participação na Classe III, segundo a análise facial. Desta forma, neste jovem há maiores expectativas de recidiva e deve-se considerar a possibilidade de um tratamento orto-cirúrgico ao término do seu crescimento.
Uma vez que o crescimento pós-tratamento não pode ser previamente determinado, torna-se imprescindivel a sobrecorreção do trespasse horizontal e a manutenção da contenção por período adequado. Isto deve ser esclarecido aos pais e ao paciente desde o início, para que eles estejam devidamente informados e convidados a cooperar com o tratamento.

\section{CONCLUSÃO}

Ao realizarmos o tratamento precoce da Classe III esquelética, devemos ser realistas. Os resultados poderão perpetuar-se ou a estabilidade será ameaçada com o retorno do padrão de crescimento original. Todos os antídotos contra a recidiva devem ser utilizados: a sobrecorreção do trespasse horizontal, o estabelecimento de um trespasse vertical adequado para o travamento da relação sagital entre os arcos dentários, e a contenção ortopédica por período prolongado. Ainda, faltaria à Ortodontia o controle da genética humana. Portanto, é importantíssima a conscientização dos pais e pacientes desde o início do tratamento, mantendo-os sempre como nossos cúmplices nessa "tentativa" de tratamento precoce.

Enviado em: Outubro de 2003 Revisado e aceito: Agosto de 2005

Orthopedical treatment of Class III in different facial patterns

\begin{abstract}
The Class III malocclusion, essentially of skeletal origin, produces an accentuated facial deformity. These malocclusion can be intercepted during the growth phase and craniofacial development by the use of orthopedical appliances. The mandibular growth, predominantly endocondral at the condilar cartilage, follows a genetic control essentially. Therefore, the effect of orthopedical appliances that aim restriction in the jaw's growth are shown limited, what leads to a poor prognostic of precocious treatment of the Class III determined by a mandibular prognathism. Fortunately, the skeletal maxillary component answers better to the application of orthopedical forces, since the growth of intramembranous bone is shown more susceptible to extrinsic influences. This way, the Class III morphologically defined by the maxillary retrognatism is privileged with the orthopedical treatment. This work discuss the interceptive treatment of Class III through the maxillary protraction in patients with different facial patterns, through the presentation of two clinical cases, and still focuss on the concerning aspects of its stability.
\end{abstract}

Key words: Facemask therapy. Rapid maxillary expansion. Skeletal prognatism. Mesiocclusion. 


\section{REFERÊNCIAS}

1. CAPELOZZA FILHO, L. et al. Tratamento Ortodôntico da Classe III: Revisando o Método (ERM e Tração) por meio de um caso clínico. R Dental Press Ortodon Ortop Facial, Maringá, v. 7, n. 6, p. 99-119, nov./dez. 2002.

2. COZZANI, G. Extraoral traction and Class III treatment. Am J Orthod Dentofacial Orthop, St. Louis, v. 80, no. 6, p. 638-650, Dec. 1981

3. ELLIS, E.; McNAMARA JR., J. A. Components of adult Class III malocclusion. J Oral Maxillofac Surg, Philadelphia, v. 42, no. 5, p. 295-305, May 1984

4. ENLOW. Crescimento facial. São Paulo: Artes Médicas, 1993.

5. FAEROVIG, E.; ZACHRISSON, B. U. Effects of mandibular incisor extraction on anterior occlusion in adults with Class III malocclusion and reduced overbite. Am J Orthod Dentofacial Orthop, St. Louis, v. 115, no. 2, p. 113-124, Feb. 1999

6. GALLAGHER, R. W.; MIRANDA, F.; BUSCHANG, P. H. Maxillary protraction: treatment and posttreatment effects. Am J Orthod Dentofacial Orthop, St. Louis, v. 113, no. 6, p. 612-619, June 1998.

7. HAAS, A. J. Rapid expansion of maxillary dental arch and nasal cavity by opening the midpalatal suture. Angle Orthod, Appleton, v. 31, no. 2, p. 73-90, Apr. 1961

8. HICKHAM, J. H. Maxillary protraction therapy: diagnosis and treatment. J Clin Orthod, Boulder, v. 25, no. 2, p. 102-113, 1991.

9. ISHII, H. et al. Treatment effect of combined maxillary protraction and chincap appliance in severe skeletal Class II cases. Am J Orthod Dentofacial Orthop, St. Louis, v. 92, no. 4, p. 304312, Oct. 1987.

10. JANSON G. R. P. et al. Tratamento e stabilidade da má oclusão de Classe III. R Dental Press Ortodon Ortop Facial, Maringá, v. 7, n. 3, p. 85-94, maio/jun. 2002

11. KAPUST, A. J.; SINCLAIR, P. M.; TURLEY, P. K. Cephalometric effects of face mask/expansion therapy in Class III children: a comparison of three age groups. Am J Orthod Dentofacial Orthop, St. Louis, v. 113, no. 2, p. 204-212, Feb. 1998.

12. MaCDONALD, K. E.; KAPUST, A. J.; TURLEY, P. K. Cephalometric changes after the correction of Class III maloccluison with maxillary expansion/facemask therapy. Am J Orthod Dentofacial Orthop, St. Louis, v. 116, no.1, p. 13-24, July 1999.

13. MCNAMARA JR., J. A. An orthopedic approach of the treatment of Class III malocclusion in young patients. J Clin Orthod, Boulder, v. 21, no. 9, p. 598-608, Sept. 1987.

14. MARTINS, D. R. et al. Tratamento da má oclusão de Classe III com máscara de protração maxilar (tração reversa). Odonto Master: Ortodontia, São Paulo, v. 1, n. 1, p. 1-10, 1994.

15. MARTINS, D. R. et al. Atlas de crescimento craniofacial. São Paulo: Ed. Santos, 1998.
16. MAZZIEIRO, E. T. et al. Aplicação da tração reversa como procedimento coadjuvante nos tratamentos ortodônticos corretivos: apresentação de um caso clínico. Ortodontia, São Paulo, v. 28, n. 1, p. 98-107, jan./abr. 1995

17. MAZZIEIRO, E. T. et al. Aplicação da tração reversa como procedimento coadjuvante nos tratamentos ortodônticos corretivos. Apresentação de um caso clínico. Ortodontia, São Paulo, v. 28 n. 1, p. 98-107, jan./abr. 1995.

18. SAADIA, M.; TORRES, E. Sagittal changes after maxillary protraction with expansion in Class III patients in the primary, mixed and late mixed detitions: A longitudinal retrospective study. Am J Orthod Dentofacial Orthop, St. Louis, v. 117, no. 6 , p. 669-680, June 2000

19. SILVA FILHO, O. G. et al. Expansão rápida da maxila na dentadura permanente: avaliação cefalométrica. Ortodontia, São Paulo, v. 25, p. 69-76, 1994.

20. SILVA FILHO, O. G.; MONTES, L. A. P.; TORELLY, L. F. Rapid maxillary expansion in the dentition evaluated posteroanterior cephalometric analysis. Am J Orthod Dentofacial Orthop St. Louis, v. 107, no. 3, p. 268-275, 1995a.

21. SILVA FILHO, O. G.; SANTOS, S. C. B. N.; SUGUIMOTO, R. M. Má oclusão de classe III: época oportuna de tratamento. Ortodontia, São Paulo, v. 28, n. 3, p. 74-84, set./dez. 1995b.

22. SILVA FILHO, O. G.; MAGRO, A. C.; OZAWA, T. O. Má oclusão de Classe III: caracterização morfológica na infância (dentaduras decídua e mista). Ortodontia, São Paulo, v. 30, n. 2, p. 7-20 maio/ago. 1997

23. SILVA FILHO, O. G.; MAGRO, A. C.; CAPELOZZA FILHO, L. Early treatment of Class III malocclusion with rapid maxillary expansion and maxillary protraction. Am J Orthod Dentofacial Orthop, St. Louis, v. 113, no. 2, p. 196-203, Feb. 1998a.

24. SILVA FILHO, O. G.; CAPELOZZA FILHO, L. Expansão rápida da maxila: preceitos clínicos. Ortodontia, São Paulo, v. 21, n. 1 p. 61-81, jan. 1998b.

25. SMALLEY, W. M. et al. Osseointegrated titanium implants for maxillofacial protraction in monkeys. Am J Orthod Dentofacial Orthop, São Paulo, v. 94, p. 285-295, 1988.

26. TAHMINA, K.; TANAKA, E.; TANNE, K. Craniofacial morphology in orthodontically treated patients of Class III malocclusion with stable and unstable treatment outcomes. Am J Orthod Dentofacial Orthop, St. Louis, v.117, no. 6, p. 681-690, June 2000.

27. TANNE, K.; HIRAGA, J.; SAKUDA, M. Effects of directions of maxillary protraction forces on biomechanical changes in craniofac complex. Eur J Orthod, London, v. 11, p. 382-391, 1989.

28. TANNE, K.; SAKUDA, M. Biomechanical and clinical changes of the craniofacial complex from orthopedic maxillary protraction. Angle Orthod, Appleton, v. 61, p. 145-152, 1991.

29. TINDLUND, R. S. Orthopedic protraction of the midface in deciduos dentition. J Craniomaxillof Surg, Philadelphia, v.17, p.17-19, 1989.

30. TURLEY, P. K. Orthopedic correction of Class III maloccusion with palatal expansion and custom protraction headgear. J Clin Orthod, Boulder, v. 22, no. 5, p. 314-324, May 1988.

\section{Endereço para correspondência}

Paula Vanessa Pedron Oltramari

Departamento de Odontopediatria, Ortodontia e Saúde Coletiva

Faculdade de Odontologia de Bauru - USP.

Alameda Dr. Octávio Pinheiro Brisolla, 9-75.

CEP: 17.043-101 - Bauru/SP - Brasil

E-mail: pvpo@usp.br 